\title{
Can Endoscopic Ultrasound or Magnetic Resonance Cholangiopancreatography Replace ERCP in Patients With Suspected Biliary Disease? A Prospective Trial and Cost Analysis
}

James M. Scheiman, M.D., F.A.C.G., Ruth C. Carlos, M.D., Jeffrey L. Barnett, M.D., F.A.C.G., Grace H. Elta, M.D., F.A.C.G., Timothy T. Nostrant, M.D., F.A.C.G., William D. Chey, M.D., F.A.C.G., Issac R. Francis, M.D., and Partha S. Nandi, M.D.

Division of Gastroenterology, Department of Internal Medicine, and Department of Radiology, University of Michigan Medical Center, Ann Arbor, Michigan

OBJECTIVES: ERCP is the gold standard for pancreaticobiliary evaluation but is associated with complications. Less invasive diagnostic alternatives with similar capabilities may be cost-effective, particularly in situations involving low prevalence of disease. The aim of this study was to compare the performance of endoscopic ultrasound (EUS) with magnetic resonance cholangiopancreatography (MRCP) and ERCP in the same patients with suspected extrahepatic biliary disease. The economic outcomes of EUS-, MRCP-, and ERCP-based diagnostic strategies were evaluated.

METHODS: Prospective cohort study of patients referred for ERCP with suspected biliary disease. MRCP and EUS were performed within $24 \mathrm{~h}$ before ERCP. The investigators were blinded to the results of the alternative imaging studies. A cost-utility analysis was performed for initial ERCP, MRCP, and EUS strategies for these patients.

RESULTS: A total of 30 patients were studied. ERCP cholangiogram failed in one patient, and another patient did not complete MRCP because of claustrophobia. The final diagnoses $(\mathrm{N}=28)$ were $\mathrm{CBD}$ stone (mean $=4 \mathrm{~mm}$; range $=$ 3-6 mm) in five patients; biliary stricture in three patients, and normal biliary tree in 20 . Two patients had pancreatitis after therapeutic ERCP, one after precut sphincterotomy followed by a normal cholangiogram. EUS was more sensitive than MRCP in the detection of choledocolithiasis (80\% vs 40\%), with similar specificity. MRCP had a poor specificity and positive predictive value for the diagnosis of biliary stricture $(76 \% / 25 \%)$ compared to EUS (100\%/ $100 \%$ ), with similar sensitivity. The overall accuracy of MRCP for any abnormality was $61 \%(95 \% \mathrm{CI}=0.41-0.78)$ compared to $89 \%$ (CI $=0.72-0.98)$ for EUS. Among those patients with a normal biliary tree, the proportion correctly identified with each test was $95 \%$ for EUS and $65 \%$ for

Presented in part as an abstract at the American Society of Gastrointestinal Endoscopy Plenary Session, Orlando, FL, May, 1999.
MRCP $(p<0.02)$. The cost for each strategy per patient evaluated was \$1346 for ERCP, \$1111 for EUS, and \$1145 for MRCP.

CONCLUSIONS: In this patient population with a low disease prevalence, EUS was superior to MRCP for choledocholithiasis. EUS was most useful for confirming a normal biliary tree and should be considered a low-risk alternative to ERCP. Although MRCP had the lowest procedural reimbursement, the initial EUS strategy had the greatest costutility by avoiding unnecessary ERCP examinations. (Am J Gastroenterol 2001;96:2900-2904. (c) 2001 by Am. Coll. of Gastroenterology)

\section{INTRODUCTION}

Endoscopic retrograde cholangiopancreatography (ERCP) remains the gold standard for evaluation of the pancreaticobiliary tree. The major drawback of this invasive procedure is the potential for serious complications. The most common serious and potentially life-threatening complications of ERCP are pancreatitis and cholangitis, which occur respectively in $5.4 \%$ and $1.0 \%$ of patients undergoing biliary sphincterotomy, even in expert centers (1). Although pancreatitis risk is increased with therapeutic ERCP, it may also occur in the setting of diagnostic procedures. Although the therapeutic potential of ERCP is unsurpassed by alternative imaging procedures, less invasive diagnostic alternatives with similar capabilities, such as endoscopic ultrasound (EUS) and magnetic resonance cholangiopancreatography (MRCP), can be considered potential alternative imaging techniques.

Endoscopic ultrasound provides high quality images of the pancreas and biliary tree. Prospective studies have supported the accuracy of EUS compared to ERCP in the diagnosis of pancreatic and biliary conditions; in fact, in highly experienced hands, EUS has been found to be more sensitive than ERCP for choledocholithiasis (2). The principal limitations of EUS are that it is an invasive procedure, 
its results are highly operator dependent, and procedure is not widely available in clinical practice. In addition, pathology identified at the time of EUS may require a subsequent therapeutic ERCP. Despite these limitations, decision analysis has supported initial EUS as opposed to ERCP as a screening tool in patients who have a low likelihood of choledocholithiasis before laparoscopic cholecystectomy (3). Even with both clinical and economic support for this strategy, this approach is not commonly used in current practice, given the practical considerations noted above.

Magnetic resonance cholangiopancreatography has emerged as a potential noninvasive alternative approach to evaluate the pancreaticobiliary system (4). Although the equipment is costly and is still under development, the lack of need for routine sedation, intravenous contrast, radiation exposure, and greater availability than EUS render MRCP an important alternative to diagnostic ERCP. Claustrophobia remains the major barrier to completion of the study. MRCP has been compared to ERCP with impressive results for tumor imaging (5); more variable results are seen in the setting of benign strictures and stones $(4,6)$. These variable results suggest that, as with EUS, the accuracy of MRCP is dependent on experience in image interpretation as well as on MR imaging techniques.

Although EUS and MRCP have compared favorably to diagnostic ERCP in detecting extrahepatic biliary disease, no study has directly compared the accuracy and clinical utility of all three modalities in the same patients. We hypothesized that these techniques may represent a viable alternative to ERCP if they can demonstrate similar accuracy and fewer complications. Thus, we prospectively evaluated EUS and MRCP in patients referred for ERCP, analyzing the performance characteristics of each test and the clinical and economic impact of using each study as the initial imaging procedure.

\section{MATERIALS AND METHODS}

\section{Patient Selection}

Consecutive adult patients ( $>18$ yr) who were referred and scheduled for ERCP on the basis of clinical signs (abnormal liver enzymes, abnormal transcutaneous ultrasound) and symptoms (biliary pain) of biliary disease were recruited. Patients with cholangitis, hypotension, or previously documented claustrophobia were excluded. For patients to be eligible for the study, EUS and MRCP had to be performed within $24 \mathrm{~h}$ before the ERCP. The University of Michigan Institutional Review Board for Human Studies approved the protocol, and patients provided written informed consent.

\section{EUS Examination Technique}

The Olympus (Tokyo, Japan) EUM-20 echoendoscope was used. After conscious sedation, the echoendoscope was placed in the duodenum and the extrahepatic biliary tract was scanned from the duodenum upon instrument withdrawal. The biliary tree was scanned from the papilla with demonstration of a "stack sign"(where bile duct and pancreatic duct are visualized parallel to each other in the pancreatic head) proximally to the hepatic hilum, if possible (7). An experienced staff endosonographer performed and interpreted the study with a trainee. This endosonographer was blinded to the results of the MRCP and ERCP.

\section{MRCP Technique}

A 1.5-T magnet was used for all imaging (Signa, GE Medical Systems, Milwaukee, WI). The body coil was used for signal transmission and reception. Three pulse sequences comprised the examination. An axial T-weighted localizer was acquired. A fast spin echo, T2-weighted sequence in the axial plane provided an overview of the upper abdomen. The MRCP sequences were comprised of multiple contiguous images centered over the central biliary tree and obtained in coronal and oblique planes. The sequence is a single-shot, fast spin echo sequence that allowed an acquisition time of 2 s/image, a total of 15 images/plane. Sequence parameters were as follows: effective $\mathrm{T} 185$, bandwidth $31.2 \mathrm{kHz}$, field of view $40 \mathrm{~cm}$, slice thickness $5.0 \mathrm{~mm}$, 0 spacing, interleaved acquisition, matrix $256 \times 256$, number of excitations 0.50 , fat saturation pulse on. Images were reviewed on a computer workstation (Advantage Windows, GE Medical Systems) using multiple operator-defined reformations and maximum intensity projection (MIP) images. The MRCP was analyzed by an experienced radiologist who was blinded to the results of the EUS and ERCP.

\section{ERCP Technique}

The ERCP was performed using a standard Pentax (Orangeburg, NY) duodenoscope by an experienced staff biliary endoscopist assisted by a trainee. The operators were blinded to the results of the EUS and MRCP when the images were interpreted.

\section{Interpretation of Imaging Tests}

The common bile duct (CBD) was interpreted as dilated if it measured $>6 \mathrm{~mm}$ in patients with intact gallbladders $(>8$ $\mathrm{mm}$ if the patient was postcholecystectomy) by EUS and MRCP, or $>10 \mathrm{~mm}$ by ERCP ( $>12 \mathrm{~mm}$ postcholecystectomy). The presence, size, and number of stones were recorded. Strictures, masses, and their locations were identified. All endoscopists and radiologists participating in the study recorded their findings independently on a standardized data form.

\section{Data Analysis}

Descriptive statistics were used to analyze the performance characteristics of each diagnostic procedure. Confidence intervals were calculated for the overall accuracy of EUS and MRCP. To compare the proportion of patients without disease correctly identified, the $\chi^{2}$ test with Yates correction was employed. Data were analyzed on a per-protocol basis. 
Table 1. Medicare Reimbursement Rates (Year 2000)

\begin{tabular}{lccr}
\hline & $\begin{array}{c}\text { Professional } \\
(\$)\end{array}$ & $\begin{array}{c}\text { Facility } \\
(\$)\end{array}$ & $\begin{array}{c}\text { Total } \\
(\$)\end{array}$ \\
\hline ERCP (diagnostic) & 428 & 511 & 939 \\
ERCP (therapeutic) & 511 & 511 & 1058 \\
EUS & 333 & 347 & 680 \\
MRCP* & & & 407 \\
Emergency room visit* & 75 & 125 & 175 \\
Transcutaneous ultrasound & & & \\
Hospitalization-DRGs & 5227 & & \\
Pancreatitis (uncomplicated) & 5774 & & \\
Cholangitis (uncomplicated) & 2345 & & \\
GI bleeding & & & \\
$\quad$ (uncomplicated) & & & \\
* Total charge not separated into professional and facility components. &
\end{tabular}

\section{Cost Analysis}

An analysis of total costs per patient diagnosed was performed. This analysis used actual Medicare procedural reimbursement rates for the year 2000 as a measure of cost. We included initial "less invasive" procedure costs and subsequent ERCP costs only if they would have been required based on the final diagnosis. Disease management costs included those associated with true positive, falsepositive, and false-negative results. For the ERCP group, the cost per patient included all observed episodes of postERCP pancreatitis. No bleeding occurred in this group. For the initial EUS and MRCP strategies, we assumed ERCP was associated with a pancreatitis rate of $5.4 \%$ (1). Because no bleeding occurred in our ERCP cases, we did not include an ERCP associated bleeding risk. EUS was assumed to have a rate of bleeding of $0.1 \%$. Modeling the outcomes of patients with missed stones and strictures is more problematic. We assumed that, in addition to the need for eventual ERCP, $80 \%$ of patients developed symptoms requiring an emergency department visit with transcutaneous ultrasound, and 10\% developed biliary colic/cholangitis requiring admission. The cost per procedure and hospitalization (Diagnosis-Related Group [DRG]) was taken from 2000 Medicare reimbursement data and is shown in Table 1.

\section{RESULTS}

During the study, 30 patients were recruited. There was one failed ERCP cholangiogram and one failed MRCP related to claustrophobia. Complete data were available for 28 patients (14 male, 14 female); these data comprise the per-protocol evaluation. The mean age of the patients studied was $46.5 \mathrm{yr}$, with a range of $23-68 \mathrm{yr}$. There were five patients with choledocholithiasis, three with biliary strictures, and 20 with a normal biliary tree. In the patients with strictures, two were secondary to a pancreatic mass and one was caused by primary sclerosing cholangitis. Two patients experienced post-ERCP pancreatitis. One of these patients had a precut sphincterotomy performed because of a strong suspicion of stones. The subsequent
Table 2. Performance of EUS and MRCP for Detection of Choledocholithiasis $(\mathrm{n}=5)$

\begin{tabular}{lcccc}
\hline & Sensitivity & Specificity & PPV & NPV \\
\hline EUS & 80 & 95 & 80 & 96 \\
MRCP & 40 & 96 & 66 & 88 \\
\hline
\end{tabular}

cholangiogram found no stones. This outcome points out both the clinical and economic importance of a treatment strategy that limits use of the highest-risk test only to those patients with disease.

The performance characteristics of EUS and MRCP for the detection of choledocholithiasis are summarized in Table 2. EUS was superior to MRCP in detecting CBD stones and approached the sensitivity of ERCP. EUS had one false-positive and one false-negative study, whereas MRCP had three false-negative studies and one false-positive. All of the patients had small stones (mean $=4 \mathrm{~mm}$, range $=$ 3-6 mm).

The performance characteristics of MRCP and EUS in the diagnosis of biliary strictures are summarized in Table 3. ERCP was superior to both EUS and MRCP in detecting biliary strictures. EUS was associated with one false-negative study (in the patient with PSC), and MRCP with one false-negative study and six false-positives. The overall accuracy of MRCP for any abnormality was 61\% (95\% $\mathrm{CI}=0.41-0.78)$ compared to $89 \%(\mathrm{CI}=0.72-0.98)$ for EUS.

Because the specificity of EUS and MRCP are most critical in defining those patients without disease, who do not need ERCP, we compared the proportion correctly identified as free of disease with each test and found that the difference was significant ( $p<0.02$ by $\chi^{2}$ test) (Fig. 1).

In evaluating the cost-utility of the initial EUS and MRCP based strategies compared with initial ERCP, EUS was associated with the lowest disease management cost (Table 4). Despite the low reimbursement for MRCP compared to EUS, in our population the use of EUS for the initial study was associated with the lowest overall cost per patient evaluated, followed by MRCP and then by ERCP. This was related to the downstream costs associated with the failure to detect stones and the incorrect identification of strictures, which prompted additional, otherwise avoidable ERCPs.

\section{DISCUSSION}

In our cohort, when compared with initial ERCP or MRCP, EUS was the most cost-effective initial imaging modality for patients with extrahepatic disease. Our study, by its

Table 3. Performance of EUS and MRCP for Evaluation of Biliary Strictures $(n=3)$

\begin{tabular}{lccrc}
\hline & Sensitivity & Specificity & PPV & NPV \\
\hline EUS & 67 & 100 & 100 & 96 \\
MRCP & 67 & 76 & 25 & 95
\end{tabular}




\section{Normal Biliary Tree}

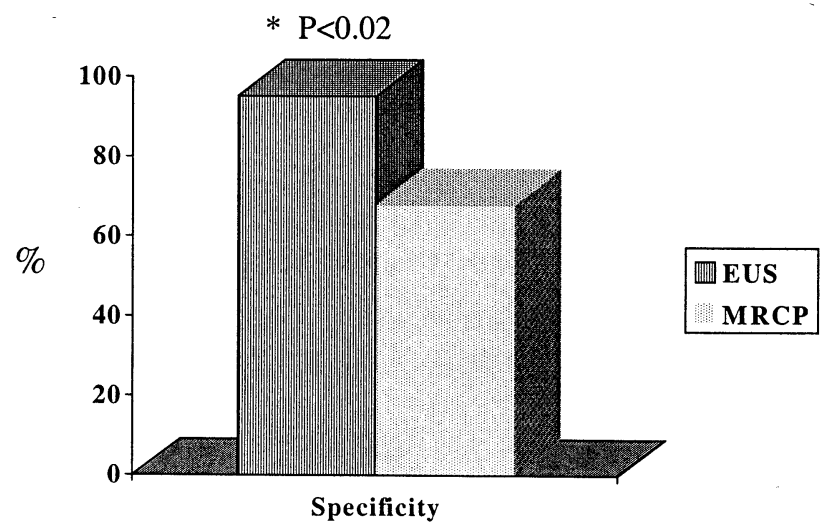

Figure 1. Specificity of EUS and MRCP for identifying a normal biliary tree. Among those patients without biliary pathology, a significantly greater proportion of cases were correctly identified with EUS compared to MRCP.

ambitious design requiring both less invasive tests to be completed within $24 \mathrm{~h}$, excluded (from a practical standpoint) patients with the need for urgent ERCP, biasing the study to include many patients with a normal biliary tree. Our observation regarding the clinically important difference in overall accuracy of the two tests $(61 \%$ vs $89 \%)$ can therefore be generalized only to populations with a low disease prevalence. Despite the overlap in $95 \% \mathrm{CI}$, supporting the need for a larger study to confirm these findings, the differences in specificity among the patients without disease had both clinical and economic impacts. By performing a simultaneous comparison of both MRCP and EUS, our unusual design does provide information previously unavailable in the literature. However, because of the relatively small number of patients studied and the low prevalence of disease, the study cannot provide broad conclusions regarding the overall diagnostic performance of EUS and MRCP.

With regard to EUS, our study supports previous data demonstrating a very high sensitivity and specificity for choledocholithiasis. In a recent decision analysis by Sahai $e t$ al. (3), a comprehensive review of the literature summarizing prospective studies of EUS for the detection of bile duct stones led to weighted averages of a sensitivity of $92 \%$ and a specificity of $98 \%$. Our data are consistent with prior literature, with our reduced sensitivity reflecting a single missed stone (one in five) in a population with a low prevalence of disease. These investigators concluded that if

Table 4. Cost-Utility Analysis

\begin{tabular}{lc}
\hline & Cost per Patient Evaluated (\$) \\
\hline EUS & 1111 \\
MRCP & 1145 \\
ERCP & 1346 \\
\hline
\end{tabular}

expert EUS was available, only high-risk patients ( $>55 \%$ likelihood of stones) should have ERCP as the initial test. Given our low disease prevalence, the cost-analysis of our prospective cohort study provides real-world outcome data to support the results of the previously mentioned economic model. The fact that a patient with suspected biliary stone disease developed a sphincterotomy complication after failure of traditional cannulation techniques only emphasizes the potential clinical and economic value of EUS before ERCP in low-risk patients, given its high specificity in patients with a normal biliary tree.

We found that EUS and MRCP provided adjunctive information for the two patients with biliary obstruction related to masses, but were poor for delineating benign biliary strictures such as those arising from primary sclerosing cholangitis. The value of EUS in detecting and staging small pancreatic tumors is unquestioned; however, ERCP retains value in its diagnostic and, most importantly, its therapeutic potential both to sample the stricture by cytology and to relieve obstruction with stenting techniques (8). In our study patients, the cause of the obstruction was not determined by traditional imaging tests such as transcutaneous ultrasound or computed tomographic scanning. Thus, the referring physician requested ERCP for primarily diagnostic purposes. Our results suggest that use of EUS or MRCP is reasonable as the initial test in this situation, and supports ERCP as the initial test when a stricture is likely, given the therapeutic and diagnostic benefits of this technique.

In our study, a major deficiency of MRCP was in the diagnosis of small biliary stones. As our patients were symptomatic, the value of diagnosis and treatment seems clear, however, there is controversy in the literature regarding the natural history of asymptomatic small stones (9), such as those found at the time of laparoscopic cholecystectomy. Although prospective outcome data would be required, in our study MRCP missed stones in three of five patients, and our economic analysis assumed, at $1 \mathrm{yr}$, that the majority (90\%) would have symptoms, with $10 \%$ requiring hospitalization. Because the patients in this study were referred for ERCP, they are not representative of the asymptomatic stone population and are likely to be at greater risk for both cholangitis and pancreatitis. The failure of MRCP to detect small stones has also been confirmed by other investigators (6).

We found that MRCP was not specific in the diagnosis of biliary strictures. This problem may lead to further diagnostic testing in patients with a normal biliary tree. The significant downstream clinical and economic costs, as reflected in our cost analysis, negated the lower cost associated with the procedure. This would further support the superiority of EUS in this clinical situation (100\% specificity) on both clinical and economic grounds, despite its invasive nature. MRCP also could not be completed in one patient because of claustrophobia. An "intent to treat" analysis, although not appropriate for this study, would worsen the performance 
characteristics of the MRCP if this patient were considered an imaging failure.

The MRCP results in the literature are quite variable, and our results are well within the range of previous reports $(6$, $10-13)$. Such variability likely reflects differences in both equipment and expertise. These factors may have played a role in this study performed in 1998-1999. Although the endosonographers had a minimum of $4 \mathrm{yr}$ experience, the radiologists had less experience with MRCP, despite their considerable MR body imaging experience. The more recent MR literature suggests innovations in technology may also lead to better diagnostic accuracy, as seen in recent studies largely outside of the United States $(11,12)$. Using the performance characteristics for EUS and MRCP in this study, our cost analysis determined that EUS was the preferred initial diagnostic test, yielding an average cost of $\$ 1111$ per patient evaluated. However, the average cost per patient for $\mathrm{MRCP}$ - a noninvasive test-was $\$ 1145$. Given the small cost difference, modest improvements in MRCP sensitivity and specificity may result in MRCP as the preferred initial test in the evaluation of individuals with suspected biliary disease. Furthermore, although Medicare reimbursement is typically used as an estimate for such cost comparisons, the true cost of procedures may differ substantially from Medicare reimbursement. This is a relevant consideration when applying our results to other health care systems. Because actual clinical practice varies to the same degree as the literature, the current cost analysis provides a framework for performing similar economic analyses for each practice. Prospective studies such as this one, although challenging to perform, will likely be needed as MRCP technology improves.

The failure of efforts to reduce ERCP pancreatitis mandates the development of less invasive alternatives such as EUS and MRCP, particularly in populations with low prevalence of disease (14). The development of new EUS technologies such as the transverse linear array technology holds the potential to develop a single endoscope that can provide biliary images and allow the operator to proceed with ERCP if necessary. With such an instrument in expert hands, we predict that the benefits to the patient and health care system will be substantial.

\section{ACKNOWLEDGMENTS}

This work was supported by grants from the General Clinical Research Center of the University of Michigan and the Robert Wood Johnson Clinical Scholars Program (to Dr. Carlos).
Reprint requests and correspondence: James M. Scheiman, M.D., Associate Professor of Medicine, Director, Endoscopic Ultrasound Program, Division of Gastroenterology, Taubman Center Box 0362, University of Michigan Medical Center, Ann Arbor, MI 48109.

Received Jan. 25, 2001; accepted June 7, 2001.

\section{REFERENCES}

1. Freeman ML, Nelson DB, Sherman S, et al. Complications of endoscopic biliary sphincterotomy. N Engl J Med 1996;335: 909-18

2. Prat F, Amouyal G, Amouyal P, et al. Prospective controlled study of endoscopic ultrasonography and endoscopic retrograde cholangiography in patients with suspected commonbile duct lithiasis. Lancet 1996;347:75-9.

3. Sahai AV, Mauldin PD, Marsi V, et al. Bile duct stones and laparoscopic cholecystectomy: A decision analysis to assess the roles of intraoperative cholangiography, EUS and ERCP. Gastrointest Endosc 1999;49:334-43.

4. Soto, JA, Barish MA, Yucel EK, et al. Magnetic resonance cholangiography: Comparison with endoscopic retrograde cholangiopancreatography. Gastroenterology 1996;110:589_97.

5. Trede M, Rumstadt B, Wendl K, et al. Ultrafast magnetic resonance imaging improves the staging of pancreatic tumors. Ann Surg 1997;226:393-407.

6. Zidi SH, Prat F, Guen OL, et al. Use of magnetic resonance cholangiography in the diagnosis of choledocholithiasis: Prospective comparison with a reference imaging method. Gut 1999; 44:118-22.

7. Cannon ME, Carpenter SL, Elta GH, et al. EUS compared with CT, MRI, and angiography, and the influence of biliary stenting on staging accuracy of ampullary neoplasms. Gastrointest Endosc 1999;50:27-33.

8. Tierney WM, Fendrick AM, Hirth RA, Scheiman JM. The clinical and economic impact of alternative staging strategies for adenocarcinoma of the pancreas. Am J Gastroenterol 2000; 95:1708-13.

9. Cotton P, Sahai A. Do asymptomatic bile duct stones need to be removed? Gastrointest Endosc 1997;46:587-94.

10. Barish MA, Yucel EK, Ferrucci JT. Magnetic resonance cholangiopancreatography. N Engl J Med 1999;341:258-64.

11. Demartines N, Eisner L, Schnabel K, et al. Evaluation of magnetic resonance cholangiography in the management of bile duct stones. Arch Surg 2000;135:148-52.

12. Liu TH, Consort ET, Kawashima A, et al. The efficacy of magnetic resonance cholangiography for the evaluation of patients with suspected choledocholithiasis before laparoscopic cholecystectomy. Am J Surg 1999;178:480-4.

13. Lee MG, Lee HJ, Kim MH, et al. Extrahepatic biliary diseases: 3D MR cholangiopancreatography compared with endoscopic retrograde cholangiopancreatography. Radiology 1997;202: 663-9.

14. Chak A, Hawes RH, Cooper GS, et al. Prospective assessment of the utility of EUS in the evaluation of gallstone pancreatitis. Gastrointest Endosc 1999;49:599-604. 